\title{
Flavonoid intake from vegetables and fruits is inversely associated with colorectal cancer risk: a case-control study in China
}

\author{
Ming $\mathrm{Xu}^{1}$, Yu-Ming Chen ${ }^{1}$, Jing Huang ${ }^{1}$, Yu-Jing Fang, ${ }^{2,3}$, Wu-Qing Huang ${ }^{1}$, Bo Yan ${ }^{1}$, Min-Shan Lu ${ }^{1,4}$, \\ Zhi-Zhong Pan $^{2}$ and Cai-Xia Zhang ${ }^{1,5 *}$ \\ ${ }^{1}$ Department of Medical Statistics and Epidemiology, School of Public Health, Sun Yat-sen University, Guangzhou 510080, \\ People's Republic of China \\ ${ }^{2}$ State Key Laboratory of Oncology in South China, Department of Colorectal Surgery, Sun Yat-sen University Cancer \\ Center, Collaborative Innovation Center for Cancer Medicine, 651 Dongfeng Road East, Guangzhou 510060, \\ People's Republic of China \\ ${ }^{3}$ State Key Laboratory of Oncology in South China, Department of Experimental Research, Sun Yat-sen University Cancer \\ Center, Collaborative Innovation Center for Cancer Medicine, 651 Dongfeng Road East, Guangzhou 510060, People's \\ Republic of China \\ ${ }^{4}$ Guangzhou Women and Children's Medical Center, Guangzhou Medical University, Guangzhou 510623, People's Republic \\ of China \\ ${ }^{5}$ Sun Yat-sen Global Health Institute, Institute of State Governance, Sun Yat-sen University, Guangzhou 510275 , \\ People's Republic of China
}

(Submitted 18 March 2016 - Final revision received 27 June 2016 - Accepted 8 August 2016 - First published online 21 September 2016)

\section{Abstract}

Flavonoids may play an important role in the protective effects of vegetables, fruits and tea against colorectal cancer. However, associations between flavonoids and colorectal cancer risk are inconsistent, and a few studies have evaluated the effect of flavonoids from different dietary sources separately. This study aimed to evaluate associations of flavonoids intake from different dietary sources with colorectal cancer risk in a Chinese population. From July 2010 to December 2015, 1632 eligible colorectal cancer cases and 1632 frequency-matched controls (age and sex) completed in-person interviews. A validated FFQ was used to estimate dietary flavonoids intake. Multivariate logistical regression models were used to calculate the OR and $95 \% \mathrm{CI}$ of colorectal cancer risk after adjusting for various confounders. No significant association was found between total flavonoids and colorectal cancer risk, with an adjusted OR of 1.06 (95\% CI 0.85, 1.32) comparing the highest with the lowest quartile. Anthocyanidins, flavanones and flavones intakes from total diet were found to be inversely associated with colorectal cancer risk. Compared with the lowest quartile, the adjusted OR for the highest quartile were 0.80 (95\% CI 0.64, 1.00) for anthocyanidins, 0.28 (95\% CI 0.22 , 0.36) for flavanones and 0.54 (95\% CI 0.43, 0.67) for flavones. All subclasses of flavonoids from vegetables and fruits were inversely associated with colorectal cancer. However, no significant association was found between tea flavonoids and colorectal cancer risk. These data indicate that specific flavonoids, specifically flavonoids from vegetables and fruits, may be linked with the reduced risk of colorectal cancer.

Key words: Flavonoids: Anthocyanidins: Flavan-3-ols: Colorectal cancer: Case-control studies

Diets rich in plant-based foods such as vegetables, fruits and tea are found to reduce the risk of developing colorectal cancer ${ }^{(1,2)}$. The mechanism by which these foods exert a protective effect is unclear, but one hypothesis is the presence of potentially anticarcinogenic phytochemicals $^{(3)}$. Flavonoids are a large group of phytochemicals widely distributed in vegetables, fruits and beverages of plant origin ${ }^{(4)}$. More than 5000 plant flavonoids have been identified thus far, and studies have usually focused on six principal subclasses: anthocyanidins, flavan-3-ols, flavanones, flavones, flavonols and isoflavones. These flavonoid compounds have been demonstrated in vitro to inhibit colon cancer cell proliferation, minimise mutation, inhibit DNA oxidation, induct phase I and II metabolic enzymes, modulate cell growth signalling pathways and mediate inflammatory response ${ }^{(5,6)}$. Therefore, we assumed that flavonoids present in vegetables, fruits or tea may play an important role in the apparent protection against colorectal cancer.

Epidemiological studies about the relationship between dietary flavonoid intake and colorectal cancer are inconsistent; two reviews published in $2013^{(7,8)}$ and a Cochrane review published in $2012^{(9)}$ concluded that no clear evidence was found to support the notion that high intake of flavonoids is associated with colorectal cancer prevention. Although some studies failed to detect an association ${ }^{(10-15)}$, several casecontrol studies ${ }^{(16-21)}$ and the Iowa Women's Health prospective cohort study ${ }^{(22)}$ suggested an inverse association between high 
flavonoid intake, particularly flavonols, flavones, catechin (flavan-3-ols) and anthocyanidins, and colorectal cancer risk.

Vegetables, fruits, tea and red wine are the main dietary sources of flavonoids, with different combination of flavonoid subclasses ${ }^{(23)}$. Vegetables and fruits almost cover all subclasses of flavonoids. Tea mainly contains flavan-3-ols, flavonols, theaflavins, thearubigins and proanthocyanidins, whereas red wine mainly contains anthocyanidins, flavan-3-ols, flavonols and proanthocyanidins. The bioavailability of flavonoids also varies in different dietary sources, depending on the exact compounds of glycoside, suggesting that the protective effect of flavonoids from different dietary sources in colorectal cancer may be different ${ }^{(24)}$. Therefore, it is necessary to examine the disparate effects on colorectal cancer risk depending on different dietary sources. However, so far, only two studies have evaluated the independent associations of different dietary sources of flavonoids with colon cancer risk. One study found that the protective effects of quercetin (a subclass of flavonols) on the risk of proximal colon cancer were significant only when fruit intake or the Healthy Eating Index score was high or when tea intake was low. In addition, increased quercetin intake had no protective effects when tea intake was high ${ }^{(17)}$. Another study found that non-tea flavonol intakes may be linked with the reduced risk of developing colorectal cancer, with an OR of 0.6 (95\% CI 0.4, 1.0) in the highest quartile of non-tea flavonols intake compared with the lowest quartile ${ }^{(18)}$, suggesting that flavonoids from dietary sources other than tea may decrease colorectal cancer risk.

In addition, epidemiological studies investigating the effects of dietary flavonoids intake on colorectal cancer risk are scarce in China. It is likely that the distribution of flavonoid subclasses differs in different populations depending on the relative intakes of vegetables, fruits or tea. The typical Chinese diet is known to be high in plant-origin foods ${ }^{(25)}$. Therefore, further studies in different regions will help elicit the role of flavonoids in colorectal cancer risk.

The aim of this study was to evaluate the associations between flavonoid intake from different dietary sources and colorectal cancer risk in a Chinese population. We hypothesised that flavonoids from different dietary sources may play different roles in colorectal cancer risk.

\section{Methods \\ Study subjects}

Details of this ongoing, hospital-based, case-control study, which began in July 2010 in Guangdong province of China for the purpose of examining the relationship between lifestyle factors and colorectal cancer risk, have been reported previously $^{(26-28)}$. In brief, between July 2010 and December 2015, a total of 1822 eligible histologically confirmed incident colorectal cancer patients were identified, and 1635 were successfully interviewed from Sun Yat-sen University Cancer Center, Guangzhou, China, with a participation rate of $89.74 \%$. Among the cancer cases who completed the FFQ, we excluded those who reported very high or very low energy intakes $(<2510$ or $>14644 \mathrm{~kJ} / \mathrm{d}(<600$ or $>3500 \mathrm{kcal} / \mathrm{d})$ for women,
$<3347$ or $>17573 \mathrm{~kJ} / \mathrm{d}$ ( $<800$ or $>4200 \mathrm{kcal} / \mathrm{d}$ ) for men); finally, 1632 cases were included in our analysis.

Controls were frequency matched to cases by 5-year age group and sex. Eligibility criteria for controls were the same as that described for the cases, except that they had no history of colorectal cancer or other cancers. Control subjects were recruited from inpatients of three affiliated hospitals of Sun Yat-sen University during the same period as the case subjects. The control subjects were selected from the Department of Ophthalmology, Ear Nose Throat, Plastic and Reconstructive Surgery, and Vascular Surgery with eye disorders, ear nose throat diseases, trigeminal neuralgia, varicose veins, osteoarthritis, degenerate joint disease, orthopaedic diseases, facial paralysis and acute appendicitis. A total of 937 hospital-derived controls were identified and 815 were successfully interviewed, yielding a participation rate of $86.98 \%$. A total of 817 control subjects were recruited from apparently healthy community residents in the same cities, invited through a number of strategies such as written invitations, flyers or referrals.

This study was conducted according to the guidelines laid down in the Declaration of Helsinki. All procedures involving human subjects were approved by The Ethical Committee of School of Public Health, Sun Yat-sen University. All participants signed informed consent forms before the interview.

\section{Data collection}

All study participants completed a face-to-face interview by trained interviewers using a structured questionnaire to collect information on dietary habits and potential confounding factors. The core questionnaire collected data on social-demographic characteristics, body height and weight, active and passive smoking, alcohol and tea intakes, physical activity, family history of cancer in first-degree relatives and previous disease history for all subjects. Data on menstrual and reproductive factors were also obtained for female subjects. Relevant medical information, medical diagnosis and histological findings were abstracted from medical records. BMI was calculated by dividing weight $(\mathrm{kg})$ by height squared $\left(\mathrm{m}^{2}\right)$.

In the present study, a current smoker was defined as someone smoking at least one cigarette a day for no less than 6 consecutive months at present ${ }^{(29)}$. Subjects were categorised as having been exposed to passive smoke if they reported ever being exposed to tobacco smoke at home or at the workplace. Regular drinking was defined as alcohol consumption at least once per week for no less than 6 consecutive months during the past year; we also asked for the type of wine consumed and the frequency and amount (Liang) of wine intake per month during the past year. Regular tea drinking was defined as tea consumption at least twice per week for no less than 3 consecutive months. Data on the type (green tea, black tea, oolong tea or others) and amount (dry weight) of tea (tea leaves) consumed during the year preceding the interview as well as the current drinking status such as frequency and how many cups $(1 \mathrm{cup}=200 \mathrm{ml}$ ) of tea intake per day were collected. Postmenopausal status was defined as at least 12 months since the last menstrual cycle. In addition, physical activity was assessed on the basis of self-reported occupational, 
household and leisure-time activities. The participants were asked to describe their occupational activities during work in the past year as (1) not working or being retired, (2) mainly sitting, (3) light intensity, (4) moderate intensity or (5) vigorous intensity. Household and leisure-time activities were also categorised into light (e.g. walking), moderate (e.g. jogging, mountaineering and playing table tennis) and vigorous (e.g. running, swimming and playing football/basketball) physical activities, and data were collected on their frequency ( $\mathrm{d} /$ week) and typical duration $(\mathrm{h} / \mathrm{d})$. The mean metabolic equivalent task (MET) hour value of each activity was obtained by estimating the average of all comparable activities in the Compendium of Physical Activities ${ }^{(30,31)}$. MET-h/week were computed using the following equation: (how many days/week $\times$ how many hours/ day $\times$ MET for a specific type of activity $=$ MET-hours/week).

Food consumption data were collected using an 81-item FFQ, including eighteen questions on fresh vegetables, twelve on fruits and seven on soya products; the questionnaire also covered fruit juice, nuts and beverages with milk and chocolate. Participants were asked to provide information on the frequency of intake and portion size during the preceding 12 months before diagnosis for cases or interview for controls, which was used to calculate the average intake of each food item in grams per day. We also provided food photographs with usual intake portion sizes to help participants estimate and record the amounts of food consumed. Energy and other nutrient intakes were computed on the basis of the 2002 Chinese Food Composition Table ${ }^{(32)}$.

In this analysis, we obtained data on total food (including grains, vegetables, fruit, tea and wine) and beverage content in terms of seven subclasses of flavonoids (anthocyanidins, flavan3-ols, flavanones, flavones, flavonols, theaflavins and thearubigins, and proanthocyanidins) according to the updated flavonoid food composition database published by the US Department of Agriculture $^{(33)}$. Isoflavones intake was computed on the basis of the 2002 Chinese Food Composition Table ${ }^{(32)}$. The measure 'total flavonoids' represents the sum of these eight compounds. As the flavonoids intake from grains and wine was low in this population, the analyses of dietary sources were focused on flavonoids intake from vegetables and fruits and flavonoids intake from tea. In this database, each subclass is a summary of individual flavonoid compounds as outlined: anthocyanidins (cyanidin, delphinidin, malvidin, pelargonidin, peonidin and petunidin); flavon-3-ols $((+)$-catechin, (-)-epicatechin, (-)-epigallocatechin, (-)-epigallocatechin 3-gallate, (-)-epicatechin 3-gallate and (+)-gallocatechin); flavones (apigenin and luteolin); flavonols (isorhamnetin, quercetin, kaempferol and myricetin); flavanones (eriodictyol, hesperidin and naringenin); theaflavins and thearubigins (theaflavin, theaflavin 3-gallate, theaflavin 3'-gallate, theaflavin 3,3'-digallate, thearubigins); isoflavones (daidzein, genistein, glycitein); and proanthocyanidins. Development and validation of the FFQ have been described elsewhere, and this FFQ has been used in a previous study ${ }^{(34)}$. The energy-adjusted correlation coefficients comparing the FFQ and the six 3-d dietary records were 0.34 for anthocyanidins, 0.61 for flavan-3-ols, 0.20 for flavanones, 0.34 for flavones, 0.27 for flavonols, 0.48 for isoflavones, 0.37 for proanthocyanidins and 0.54 for total flavonoids. The correlation coefficients between the two FFQ administered 1 year apart were 0.12 for anthocyanidins, 0.51 for flavan-3-ols, 0.66 for flavanones, 0.32 for flavones, 0.54 for flavonols, 0.39 for isoflavones, 0.36 for proanthocyanidins and $0 \cdot 44$ for total flavonoids.

\section{Statistical analysis}

SPSS software (version 21.0) was used to conduct the data analysis. Flavonoids intakes and food groups were adjusted for total energy intake by the regression residual method ${ }^{(35)}$. Subjects were divided into quartiles (Q1-Q4) based on the distribution of flavonoids intakes among the control group. Continuous variables (such as age, subclasses of flavonoids intake) were compared between cases and controls using Student's $t$ test, whereas categorical variables (such as income, education level, smoking status) were analysed using $\chi^{2}$ tests. Unconditional logistic regression models were used to estimate the OR and 95\% CI for the associations of flavonoids intakes with the risk of colorectal cancer, using the lowest quartile as the reference group. The OR and $95 \%$ CI were further adjusted for potential confounding by the following variables: age, sex, marital status, education, income, occupation, family history of cancer, smoking status, passive smoking, alcohol drinking, occupational activity, household and leisure-time activities, and BMI. Intakes of red and processed meat, poultry and fish, total dairy products and eggs were statistically significantly different between cases and controls. As these foods were not sources of flavonoids, they were treated as confounders and added into the logistic regression models. Tests for trend were performed by entering categorical variables as continuous variables in the multiple regression models.

Stratified analyses by sex were conducted for the associations between flavonoids intake and colorectal cancer risk. The interaction between sex and intake of flavonoids in relation to the risk of colorectal cancer was evaluated in multiplicative models by including the product term in it. Stratified analysis by socio-economic status (according to education level and income level) was also conducted, which was not well balanced between cases and controls in the present study. Education levels were classified into four stages (primary school or below, secondary school, high school and college or above). Income levels were also classified into four stages $(<2000$, 2001-5000, 5001-8000>8001 yuan/month). Subjects with education stage plus income stage values less than 5 were defined as individuals with low socio-economic status, and otherwise defined as individuals with high socio-economic status. In addition, we conducted subgroup analyses by cancer site (colon or rectal cancer) and by sources of controls (community-derived controls and hospital-derived controls). Sensitivity analysis using only community-derived controls or hospital-derived controls was also conducted. All $P$ values were two-sided and statistical significance was determined at the $P<0.05$ level in the present study.

\section{Results}

Table 1 shows the socio-demographic characteristics of the study subjects and the distribution of selected colorectal cancer risk factors. Of the 1632 cases, 910 were males and 722 were 
Table 1. Demographic and selected risk factors of colorectal cancer cases and controls in a Chinese population* (Numbers and percentages; mean values and standard deviations; medians and 25th, 75th percentiles)

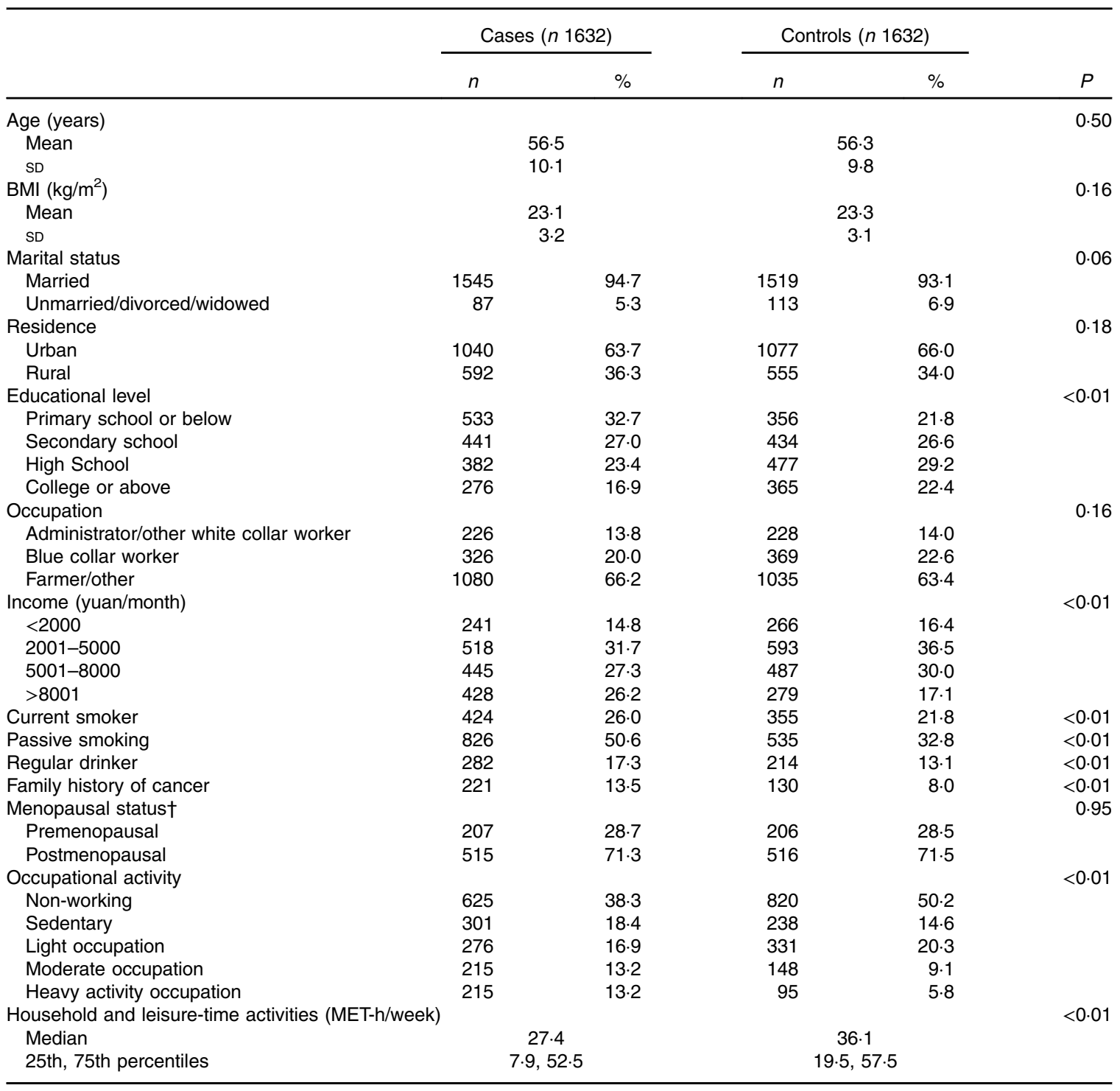

MET, metabolic equivalent task.

* Continuous variables were evaluated using $t$ tests or Wilcoxon's rank-sum tests. Categorical variables were evaluated using $\chi^{2}$ tests. † Among female subgroups.

females; 983 (567 males and 416 females) subjects were diagnosed with colon cancer and 649 (343 males and 306 females) with rectal cancer. Compared with controls, case subjects had lower education levels, greater household income, heavier occupational activity, less household and leisure-time activity and were more likely to have a family history of cancer, to be drinkers and current smokers and have passive smoking experience. All of the above variables were considered potential confounders and adjusted for in the subsequent analysis. No significant differences were found between cases and controls in other factors including marital status, occupation status, BMI and menopausal status (in female subgroup).

The comparison of intakes of flavonoid subclasses from different dietary sources between cases and controls and selected dietary confounders are shown in Table 2.
The energy-adjusted median intake of total flavonoids showed no difference between cases $(241.0 \mathrm{mg} / \mathrm{d})$ and controls $(256.5 \mathrm{mg} / \mathrm{d})$. Compared with controls, anthocyanidins, flavan3-ols, flavanones, flavones, flavonols, proanthocyanidins and total flavonoids intakes from vegetables and fruits were significantly lower in cases. The energy-adjusted median intakes of flavan-3-ols, flavonols, proanthocyanidins and total flavonoids form tea were significantly higher among cases than among controls. Moreover, cases were reported to have more red and processed meat, less poultry and fish, total dairy products and eggs compared with controls.

The main food sources of subclasses and total flavonoids among control subjects are listed in Table 3. The richest source of total flavonoids was tea $(48.94 \%)$, followed by apple (10.63\%) and grapes (8.02\%). Anthocyanidins were rich in 
Table 2. Intakes of energy, flavonoids and food groups among case and control subjects in Guangdong, China* (Mean values and standard deviations; medians and 25th, 75th percentiles)

\begin{tabular}{|c|c|c|c|c|c|c|c|c|c|}
\hline & \multicolumn{4}{|c|}{ Cases ( $n$ 1632) } & \multicolumn{4}{|c|}{ Controls ( $n$ 1632) } & \multirow[b]{2}{*}{$P$} \\
\hline & Mean & SD & Median & 25th, 75th percentiles & Mean & SD & Median & 25th, 75th percentiles & \\
\hline Energy (kJ/d) & 6770 & $2104 \cdot 6$ & 6477 & $5267 \cdot 7,7887$ & 7284 & 2269 & 6979 & 5653,8506 & \\
\hline Energy $(\mathrm{kcal} / \mathrm{d})$ & 1618 & 503.0 & 1548 & 1259,1885 & 1741 & $542 \cdot 3$ & 1668 & 1351,2033 & $<0.01$ \\
\hline \multicolumn{10}{|l|}{ Flavonoids intake from total diet† } \\
\hline Anthocyanidins (mg/d) & 20.99 & 15.68 & 17.52 & $10 \cdot 75,26 \cdot 16$ & 24.71 & 28.64 & $20 \cdot 16$ & $12 \cdot 43,30 \cdot 81$ & $<0.01$ \\
\hline Flavan-3-ols (mg/d) & $176 \cdot 80$ & $335 \cdot 40$ & $31 \cdot 28$ & $7 \cdot 35,184.50$ & $158 \cdot 60$ & $320 \cdot 70$ & $21 \cdot 82$ & $7 \cdot 27,151 \cdot 00$ & 0.08 \\
\hline Flavanones (mg/d) & 3.85 & $4 \cdot 38$ & $2 \cdot 82$ & $1.58,4.56$ & $7 \cdot 18$ & $9 \cdot 36$ & 4.08 & $2 \cdot 21,8 \cdot 27$ & $<0.01$ \\
\hline Flavones (mg/d) & 3.67 & 5.09 & $2 \cdot 21$ & $0.81,4.34$ & 4.05 & 3.84 & 2.92 & $1.49,5.28$ & $<0.01$ \\
\hline Flavonols (mg/d) & 37.67 & 19.87 & 34.50 & $23.49,46.91$ & 37.72 & 19.48 & 34.42 & $23 \cdot 71,47 \cdot 01$ & 0.80 \\
\hline Isoflavones (mg/d) & $9 \cdot 01$ & 9.63 & $6 \cdot 34$ & $2 \cdot 88,12 \cdot 14$ & 8.86 & 9.69 & 5.93 & $2 \cdot 38,12 \cdot 36$ & 0.18 \\
\hline Theaflavins and thearubigins $(\mathrm{mg} / \mathrm{d})$ & 35.33 & $102 \cdot 30$ & 0.00 & $0.00,19.83$ & 28.96 & 89.28 & 0.00 & $0.00,14.44$ & 0.69 \\
\hline Proanthocyanidins $(\mathrm{mg} / \mathrm{d})$ & $112 \cdot 30$ & $90 \cdot 79$ & 91.63 & $48.46,148.30$ & $126 \cdot 20$ & $100 \cdot 60$ & $104 \cdot 10$ & $58 \cdot 67,170.60$ & $<0.01$ \\
\hline Total flavonoids $(\mathrm{mg} / \mathrm{d})$ & $399 \cdot 10$ & $427 \cdot 10$ & 241.00 & $135.90,503.50$ & 395.00 & 414.90 & $256 \cdot 50$ & $145 \cdot 70,475 \cdot 60$ & 0.26 \\
\hline \multicolumn{10}{|c|}{ Flavonoids intake from vegetables and fruits $\dagger$} \\
\hline Anthocyanidins $(\mathrm{mg} / \mathrm{d})$ & 20.64 & $15 \cdot 25$ & $17 \cdot 30$ & $10 \cdot 71,25 \cdot 86$ & 23.60 & 16.44 & $19 \cdot 90$ & $12 \cdot 39,30 \cdot 26$ & $<0.01$ \\
\hline Flavan-3-ols (mg/d) & 7.04 & 6.73 & $5 \cdot 27$ & $2 \cdot 80,9.28$ & 8.55 & 7.07 & 6.89 & $3.82,11.30$ & $<0.01$ \\
\hline Flavanones (mg/d) & $3 \cdot 76$ & $4 \cdot 38$ & $2 \cdot 71$ & $1.51,4.44$ & $7 \cdot 11$ & $9 \cdot 35$ & 3.92 & $2 \cdot 16,8 \cdot 18$ & $<0.01$ \\
\hline Flavones $(\mathrm{mg} / \mathrm{d})$ & 3.67 & 5.09 & $2 \cdot 21$ & $0.81,4.34$ & 4.05 & $3 \cdot 84$ & 2.92 & $1.49,5 \cdot 28$ & $<0.01$ \\
\hline Flavonols $(\mathrm{mg} / \mathrm{d})$ & $30 \cdot 27$ & $16 \cdot 58$ & 27.57 & $18 \cdot 64,38.84$ & $31 \cdot 22$ & $15 \cdot 72$ & 28.56 & $20.04,39.27$ & 0.02 \\
\hline Isoflavones $(\mathrm{mg} / \mathrm{d})$ & 9.01 & 9.63 & $6 \cdot 34$ & $2 \cdot 88,12 \cdot 14$ & 8.86 & 9.69 & 5.93 & $2.38,12.36$ & 0.18 \\
\hline Proanthocyanidins (mg/d) & $109 \cdot 30$ & 88.43 & 89.00 & $47.53,143.80$ & $122 \cdot 50$ & 93.48 & $101 \cdot 20$ & $58.01,165.00$ & $<0.01$ \\
\hline Total flavonoids $(\mathrm{mg} / \mathrm{d})$ & 183.40 & $112 \cdot 10$ & $157 \cdot 90$ & $108 \cdot 40,228.60$ & $206 \cdot 20$ & 117.60 & 184.50 & $125 \cdot 20,264 \cdot 40$ & $<0.01$ \\
\hline \multicolumn{10}{|l|}{ Flavonoids intake from tea† } \\
\hline Flavan-3-ols (mg/d) & $167 \cdot 60$ & 343.50 & $21 \cdot 15$ & $0.00,179 \cdot 10$ & $158 \cdot 70$ & 338.00 & 9.83 & $0.00,146.90$ & 0.03 \\
\hline Theaflavins and thearubigins $(\mathrm{mg} / \mathrm{d})$ & $35 \cdot 33$ & $102 \cdot 30$ & 0.00 & $0.00,19.83$ & 28.96 & 89.28 & 0.00 & $0.00,14.44$ & 0.69 \\
\hline Flavonols $(\mathrm{mg} / \mathrm{d})$ & $7 \cdot 28$ & $11 \cdot 12$ & 1.80 & $0.00,11.23$ & 6.74 & $11 \cdot 27$ & 1.40 & $0.00,9.43$ & 0.02 \\
\hline Proanthocyanidins (mg/d) & $2 \cdot 16$ & 3.27 & 0.55 & $0.00,3.46$ & 2.01 & 3.43 & 0.42 & $0.00,2.76$ & $<0.01$ \\
\hline Total flavonoids $(\mathrm{mg} / \mathrm{d})$ & 211.90 & 373.90 & 61.36 & $0.00,273.20$ & $196 \cdot 50$ & 369.90 & $30 \cdot 30$ & $0.00,224.20$ & 0.02 \\
\hline Red and processed meat $(\mathrm{g} / \mathrm{d}) \dagger$ & 131.40 & 69.50 & $120 \cdot 60$ & $82.69,168.20$ & 98.87 & 54.50 & $90 \cdot 58$ & $58.38,129.40$ & $<0.01$ \\
\hline Poultry and fish $(\mathrm{g} / \mathrm{d}) \dagger$ & $88 \cdot 26$ & 74.86 & 68.94 & $39.63,112 \cdot 70$ & $104 \cdot 70$ & $75 \cdot 74$ & $85 \cdot 60$ & $51.81,137.90$ & $<0.01$ \\
\hline Total dairy products $(\mathrm{g} / \mathrm{d}) \dagger$ & 45.41 & 131.80 & 0.41 & $0.00,28.50$ & 84.27 & $177 \cdot 90$ & $20 \cdot 24$ & $0.42,92.16$ & $<0.01$ \\
\hline Eggs $(g / d) t$ & 20.53 & 17.89 & $16 \cdot 70$ & $7.64,26 \cdot 89$ & 24.11 & $20 \cdot 72$ & $20 \cdot 46$ & $10.06,33.93$ & $<0.01$ \\
\hline
\end{tabular}

* Wilcoxon's rank-sum test comparing the median intake levels between cases and controls.

$\dagger$ Adjusted for total energy intake by the regression residual method.

Table 3. Top five food sources of subclasses of flavonoids among control subjects

\begin{tabular}{|c|c|c|c|}
\hline Food sources & Proportion & Food sources & Proportion \\
\hline Total flavonoids & & Flavones & \\
\hline Tea & 48.94 & Chinese celery & $76 \cdot 17$ \\
\hline Apple & 10.63 & Peppers & 9.53 \\
\hline Grape & 8.02 & Lettuce & 3.05 \\
\hline Leaf mustard & 3.89 & Pumpkin & 1.98 \\
\hline Nuts & 3.34 & Leaf mustard & 1.48 \\
\hline Anthocyanidins & & Flavonols & \\
\hline Eggplant & $30 \cdot 26$ & Leaf mustard & 28.86 \\
\hline Grape & 23.34 & Tea & $15 \cdot 31$ \\
\hline Radish & $22 \cdot 25$ & Watercress & 13.62 \\
\hline Apple & 6.94 & Peppers & 12.59 \\
\hline Banana & 5.43 & Spinach & 4.74 \\
\hline Flavan-3-ols & & Proanthocyanidins & \\
\hline Tea & 94.10 & Apple & 34.11 \\
\hline Apple & $2 \cdot 71$ & Grape & 23.66 \\
\hline Beans & 0.87 & Nuts & 12.90 \\
\hline Banana & 0.71 & Beans & 10.53 \\
\hline Grape & 0.54 & Pear & 5.96 \\
\hline Flavanones & & Isoflavones & \\
\hline Citrus & $86 \cdot 32$ & Soya milk & 33.71 \\
\hline Leaf mustard & 8.38 & Soft tofu & $27 \cdot 79$ \\
\hline Fruit juice & 2.93 & Processed soya products & 24.08 \\
\hline White wine & 1.37 & Soyabean & $9 \cdot 70$ \\
\hline Tomato & 0.84 & Hard tofu & 4.72 \\
\hline
\end{tabular}

vegetables (such as eggplant, radish), grapes, apple and banana. Most of the flavan-3-ols were contained in tea and apple. Flavanones were rich in fruits, especially in citrus fruits or citrus juice. Flavones were also rich in vegetables such as Chinese celery, peppers and lettuce. Flavonols were rich in leaf mustard and tea. The top five sources of proanthocyanidins were apple, grapes, nuts, beans and pear. Isoflavones were mainly contained in soya products.

As shown in Table 4, significant inverse associations were observed between dietary flavanones, flavones and the risk of colorectal cancer. The multivariate OR for the highest quartile compared with the lowest quartile were 0.28 (95\% CI 0.22 , 0.36 ) for flavanones and 0.54 (95\% CI $0.43,0.67)$ for flavones. There was borderline significant inverse association between total anthocyanidins intake and colorectal cancer risk, with OR for the highest quartile compared with the lowest quartile of 0.80 (95\% CI 0.64, 1.00) ( $\left.P_{\text {trend }}=0.08\right)$. No significant association was found for flavan-3-ols, flavonols and total flavonoids from total diet.

The association between flavonoid intake from different dietary sources and colorectal cancer risk is shown in Table 5. Significant inverse associations were observed between all subclasses of flavonoid intake from vegetables and fruits and 
Table 4. Colorectal cancer according to quartiles $(Q)$ of flavonoids intake from total diet (Odds ratios and $95 \%$ confidence intervals)

\begin{tabular}{|c|c|c|c|c|c|c|c|c|}
\hline & \multirow{2}{*}{$\frac{\mathrm{Q1}}{\mathrm{OR}}$} & \multicolumn{2}{|c|}{ Q2 } & \multicolumn{2}{|c|}{ Q3 } & \multicolumn{2}{|c|}{ Q4 } & \multirow[b]{2}{*}{$P_{\text {trend }}$} \\
\hline & & OR & $95 \% \mathrm{Cl}$ & OR & $95 \% \mathrm{Cl}$ & OR & $95 \% \mathrm{Cl}$ & \\
\hline \multicolumn{9}{|l|}{ Anthocyanidins } \\
\hline No. cases/controls & $516 / 407$ & \multicolumn{2}{|c|}{$430 / 409$} & \multicolumn{2}{|c|}{$381 / 409$} & \multicolumn{2}{|c|}{$305 / 407$} & \\
\hline Crude & 1 & 0.83 & $0.69,1.00$ & 0.73 & $0.61,0.89$ & 0.59 & $0.49,0.72$ & $<0.01$ \\
\hline Adjusted* & 1 & 0.91 & $0.73,1.12$ & 0.93 & $0.75,1.15$ & 0.80 & $0.64,1.00$ & 0.08 \\
\hline \multicolumn{9}{|l|}{ Flavan-3-ols } \\
\hline No. cases/controls & $392 / 407$ & \multicolumn{2}{|c|}{$396 / 409$} & \multicolumn{2}{|c|}{$409 / 409$} & \multicolumn{2}{|c|}{$435 / 407$} & \\
\hline Crude & 1 & 1.01 & $0.83,1.22$ & 1.04 & $0.85,1.26$ & $1 \cdot 11$ & $0.91,1.35$ & 0.27 \\
\hline Adjusted* & 1 & $1 \cdot 18$ & $0.94,1.47$ & $1 \cdot 18$ & $0.95,1.48$ & 1.25 & $0.99,1.56$ & 0.07 \\
\hline \multicolumn{9}{|l|}{ Flavanones } \\
\hline No. cases/controls & $640 / 407$ & \multicolumn{2}{|c|}{$492 / 409$} & \multicolumn{2}{|c|}{$360 / 409$} & \multicolumn{2}{|c|}{$140 / 407$} & \\
\hline Crude & 1 & 0.76 & $0.64,0.92$ & 0.56 & $0.46,0.68$ & 0.22 & $0.17,0.27$ & $<0.01$ \\
\hline Adjusted ${ }^{*}$ & 1 & 0.76 & $0.62,0.92$ & 0.61 & $0.50,0.76$ & 0.28 & $0.22,0.36$ & $<0.01$ \\
\hline \multicolumn{9}{|l|}{ Flavones } \\
\hline No. cases/controls & $609 / 407$ & \multicolumn{2}{|c|}{$366 / 409$} & \multicolumn{2}{|c|}{$338 / 409$} & \multicolumn{2}{|c|}{$319 / 407$} & \\
\hline Crude & 1 & 0.60 & $0.50,0.72$ & 0.55 & $0.46,0.67$ & 0.52 & $0.43,0.64$ & $<0.01$ \\
\hline Adjusted* & 1 & 0.55 & $0.44,0.68$ & 0.54 & $0.44,0.67$ & 0.54 & $0.43,0.67$ & $<0.01$ \\
\hline \multicolumn{9}{|l|}{ Flavonols } \\
\hline No. cases/controls & $430 / 407$ & \multicolumn{2}{|c|}{$387 / 409$} & \multicolumn{2}{|c|}{$398 / 409$} & \multicolumn{2}{|c|}{$417 / 407$} & \\
\hline Crude & 1 & 0.90 & $0.74,1.09$ & 0.92 & $0.76,1.12$ & 0.97 & $0.80,1.18$ & 0.83 \\
\hline Adjusted $^{*}$ & 1 & 0.83 & $0.66,1.03$ & 0.81 & $0.65,1.00$ & 0.80 & $0.64,0.99$ & 0.05 \\
\hline \multicolumn{9}{|l|}{ Total flavonoids } \\
\hline No. cases/controls & $443 / 407$ & \multicolumn{2}{|c|}{$415 / 409$} & \multicolumn{2}{|c|}{$366 / 409$} & \multicolumn{2}{|c|}{$408 / 407$} & \\
\hline Crude & 1 & 0.93 & $0.77,1.13$ & 0.82 & $0.68,1.00$ & 0.92 & $0.76,1.12$ & 0.23 \\
\hline Adjusted* & 1 & $1 \cdot 11$ & $0.90,1.38$ & 1.04 & $0.84,1.31$ & 1.06 & $0.85,1.32$ & 0.78 \\
\hline
\end{tabular}

* OR was adjusted for age, sex, marital status, education, income, occupation, family history of cancer, smoking status, passive smoking, alcohol drinking, occupational activity, household and leisure-time activities, BMI, and intakes of red and processed meat, poultry and fish, total dairy products and eggs.

colorectal cancer risk. The multivariate OR for the highest quartile of flavonoids from vegetables and fruits compared with the lowest quartile were 0.74 (95\% CI 0.59, 0.92) for flavan-3ols, 0.28 (95\% CI 0.22, 0.36) for flavanones, 0.54 (95\% CI 0.43, $0.67)$ for flavones, $0.74(95 \%$ CI $0.59,0.92)$ for flavonols and $0.70(95 \%$ CI $0.55,0.87)$ for total flavonoids. Borderline significant inverse association was also found between anthocyanidins intake from vegetables and fruits and risk of colorectal cancer (OR 0.79; 95\% CI 0.63, 0.98, $P_{\text {trend }}=0.06$ ). However, no significant association was found between flavonoids intake from tea and colorectal cancer (OR 0.94; $95 \%$ CI 0.75, 1.17).

Consistent with the total participants, stratified analysis by sex showed that the inverse associations between intakes of flavanones and flavones and colorectal cancer risk were observed in both sexes (Table 6). No significant associations were found between intakes of flavan-3-ols and total flavonoids and risk of colorectal cancer among men and women. Although inverse associations between anthocyanidins and flavonols intakes and colorectal cancer risk were observed only among men and not women, no significant interaction was found between anthocyanidins and flavanols intakes and sex on the risk of colorectal cancer $\left(P_{\text {interaction }}=0.06\right.$ for anthocyanidins, $P_{\text {interaction }}=0 \cdot 12$ for flavonols). The results of subgroup analysis by cancer site were consistent with the main results (Table 7). The associations of flavonoids intake with colorectal cancer risk did not differ significantly when stratified by socio-economic status (low socio-economic status and high socio-economic status) (Table 8). Subgroup analysis by community-derived controls and hospital-derived controls showed no significant difference when using either group. Sensitivity analysis by using only community-derived controls or hospital-derived controls also showed that the results were relatively stable (data not shown).

\section{Discussion}

This case-control study, with 1632 cases and 1632 controls, revealed no significant association between total flavonoids intake and colorectal cancer risk. However, higher intake of anthocyanidins, flavanones and flavones from total diet was inversely associated with colorectal cancer risk. All subclasses of flavonoids from vegetables and fruits were inversely associated with colorectal cancer risk. However, tea flavonoids were not significantly associated with colorectal cancer risk.

Some previous studies have examined the association between total flavonoids intake and colorectal cancer risk, and the results are consistent with the present study ${ }^{(10,13,21)}$. A US cohort of 107401 subjects ( 71976 from the Nurses' Health Study and 35425 from the Health Professionals Follow-up Study) found that the intake of total flavonoids was not associated with colorectal cancer risk, with the relative risk (RR) for the highest quintile of total flavonoids intake being 1.19 (95\% CI 0.94, 1.49) compared with the lowest ${ }^{(10)}$. No significant association was found between total flavonoids intake and risk of colorectal cancer in Finnish men. The hazard risk for the highest quartile compared with the lowest was 1.16 (95\% CI $0.58,2 \cdot 34)^{(13)}$. The multicentric, Italian case-control study with 1953 cases and 4154 controls also found no association between total flavonoids and colorectal cancer risk; the adjusted OR for the highest 
Table 5. Colorectal cancer according to quartiles (Q) of flavonoids intake from different dietary sources (Odds ratios and $95 \%$ confidence intervals)

\begin{tabular}{|c|c|c|c|c|c|c|c|c|}
\hline & \multirow{2}{*}{$\frac{\mathrm{Q1}}{\mathrm{OR}}$} & \multicolumn{2}{|c|}{ Q2 } & \multicolumn{2}{|c|}{ Q3 } & \multicolumn{2}{|c|}{ Q4 } & \multirow[b]{2}{*}{$P_{\text {trend }}$} \\
\hline & & OR & $95 \% \mathrm{Cl}$ & OR & $95 \% \mathrm{Cl}$ & OR & $95 \% \mathrm{Cl}$ & \\
\hline \multicolumn{9}{|c|}{ Flavonoids intake from vegetables and fruits } \\
\hline \multicolumn{9}{|c|}{ Anthocyanidins } \\
\hline No. cases/controls & $514 / 407$ & \multicolumn{2}{|c|}{$432 / 409$} & \multicolumn{2}{|c|}{$382 / 409$} & \multicolumn{2}{|c|}{$304 / 407$} & \\
\hline Crude & 1 & 0.84 & $0.69,1.01$ & 0.74 & $0.61,0.89$ & 0.59 & $0.49,0.72$ & $<0.01$ \\
\hline Adjusted* & 1 & 0.91 & $0 \cdot 73,1 \cdot 12$ & 0.93 & $0 \cdot 75,1 \cdot 16$ & 0.79 & $0.63,0.98$ & 0.06 \\
\hline \multicolumn{9}{|l|}{ Flavan-3-ols } \\
\hline No. cases/controls & $603 / 407$ & \multicolumn{2}{|c|}{$399 / 409$} & \multicolumn{2}{|c|}{$328 / 409$} & \multicolumn{2}{|c|}{$302 / 407$} & \\
\hline Crude & 1 & 0.66 & $0.55,0.79$ & 0.54 & $0.45,0.66$ & 0.50 & $0.41,0.61$ & $<0.01$ \\
\hline Adjusted* & 1 & 0.80 & $0.65,0.99$ & 0.73 & $0.58,0.90$ & 0.74 & $0.59,0.92$ & $<0.01$ \\
\hline \multicolumn{9}{|l|}{ Flavanones } \\
\hline No. cases/controls & $640 / 407$ & \multicolumn{2}{|c|}{$501 / 409$} & \multicolumn{2}{|c|}{$353 / 409$} & \multicolumn{2}{|c|}{$138 / 407$} & \\
\hline Crude & 1 & 0.78 & $0.65,0.93$ & 0.55 & $0.45,0.66$ & 0.22 & $0.17,0.27$ & $<0.01$ \\
\hline Adjusted ${ }^{*}$ & 1 & 0.75 & $0.61,0.91$ & 0.62 & $0.50,0.76$ & 0.28 & $0.22,0.36$ & $<0.01$ \\
\hline \multicolumn{9}{|l|}{ Flavones } \\
\hline No. cases/controls & $609 / 407$ & \multicolumn{2}{|c|}{$366 / 409$} & \multicolumn{2}{|c|}{$338 / 409$} & \multicolumn{2}{|c|}{$319 / 407$} & \\
\hline Crude & 1 & 0.60 & $0.50,0.72$ & 0.55 & $0.46,0.67$ & 0.52 & $0.43,0.64$ & $<0.01$ \\
\hline Adjusted $^{*}$ & 1 & 0.55 & $0.44,0.68$ & 0.54 & $0.44,0.67$ & 0.54 & $0.43,0.67$ & $<0.01$ \\
\hline \multicolumn{9}{|l|}{ Flavonols } \\
\hline No. cases/controls & $478 / 407$ & \multicolumn{2}{|c|}{$381 / 409$} & \multicolumn{2}{|c|}{$369 / 409$} & \multicolumn{2}{|c|}{$404 / 407$} & \\
\hline Crude & 1 & 0.79 & $0.65,0.96$ & 0.77 & $0.63,0.93$ & 0.85 & $0.70,1.02$ & 0.07 \\
\hline Adjusted $^{\star}$ & 1 & 0.75 & $0.60,0.93$ & 0.67 & $0.54,0.83$ & 0.74 & $0.59,0.92$ & $<0.01$ \\
\hline \multicolumn{9}{|c|}{ Total flavonoids from vegetables and fruits } \\
\hline No. cases/controls & $547 / 407$ & \multicolumn{2}{|c|}{$433 / 409$} & \multicolumn{2}{|c|}{$359 / 409$} & & /407 & \\
\hline Crude & 1 & 0.79 & $0.65,0.95$ & 0.65 & $0.54,0.79$ & 0.54 & $0.44,0.65$ & $<0.01$ \\
\hline Adjusted $^{*}$ & 1 & 0.90 & $0.73,1.11$ & 0.81 & $0.65,1.01$ & 0.70 & $0.55,0.87$ & $<0.01$ \\
\hline Total flavonoids form tea & & & & & & & & \\
\hline No. cases/controls & $410 / 407$ & & 1409 & & $9 / 409$ & & $/ 407$ & \\
\hline Crude & 1 & 0.92 & $0.76,1 \cdot 12$ & 1.04 & $0.86,1.26$ & 1.00 & $0.83,1.22$ & 0.67 \\
\hline Adjusted ${ }^{*}$ & 1 & 0.93 & $0.74,1.16$ & 0.97 & $0.78,1.20$ & 0.94 & $0.75,1.17$ & 0.69 \\
\hline
\end{tabular}

* OR was adjusted for age, sex, marital status, education, income, occupation, family history of cancer, smoking status, passive smoking, alcohol drinking, occupational activity, household and leisure-time activities, BMI, and intakes of red and processed meat, poultry and fish, total dairy products and eggs.

quintile compared with lowest was $0.97(95 \% \text { CI } 0 \cdot 81,1 \cdot 16)^{(21)}$. However, the Spanish case-control study with 424 cases and 401 controls found an inverse association between total flavonoids intake and colorectal cancer risk (OR 0.59; $95 \% \mathrm{CI}$ $0.35,0.99$ for the highest quintile $v$. the lowest ${ }^{(16)}$. Although it was reported that total flavonoid intake may be greatly influenced by thearubigins, particularly in high black tea-consuming countries ${ }^{(36)}$, the present study found no significant difference between total flavonoids intake and colorectal cancer risk before and after thearubigins were excluded from total flavonoid intake. This result is consistent with two other studies that did not include thearubigins in total flavonoid intake ${ }^{(16,21)}$. Different results between total flavonoids intake and colorectal cancer risk in different studies could be partly explained by the variability in flavonoid sources. The participants of the Spanish case-control study were reported to consume relatively large amounts of flavonoids from vegetables and fruits and the flavonoids from tea was $<10 \%{ }^{(16)}$, whereas tea was a major single food source of flavonoids ( $35 \%$ contribution) in the US cohort ${ }^{(10)}$. It is likely that the distribution of flavonoid subclasses differs in different dietary resources. Examining flavonoids from total diet and colorectal cancer without taking into account the different sources may have contributed to the null result. If the effect of flavonoids from vegetables and fruits is contrary to that from tea, then the effect of the combined flavonoids from vegetables, fruit and tea together will be weakened.

Intake of anthocyanidins, flavanones and flavones from total diet was inversely associated with colorectal cancer risk in the present study. The Spanish case-control study observed a $41 \%$ reduction in colorectal cancer risk in the highest quintile of flavones intake ${ }^{(16)}$. A reduced risk of colorectal cancer was found for high intake of anthocyanidins (OR 0.67, the highest $v$. the lowest quintile), flavonols (OR 0.64, the highest $v$. the lowest quintile), flavones (OR $0 \cdot 78$, the highest $v$. the lowest quintile) in a network of case-control studies from Italy $^{(19)}$. Another Scottish case-control study with 1456 cases and 1456 controls found reductions in colorectal cancer risk associated with intake of flavonols and its main compound quercetin ${ }^{(20)}$. The multicentric, Italian, case-control study also found a reduced risk of developing colorectal cancer with increasing intake of anthocyanidins, flavones and flavonols ${ }^{(21)}$. Although the protective effect of flavanones on colorectal cancer risk has not been observed in previous studies, in vitro studies have shown that flavanones can induce apoptosis, inhibit cancer growth and have anti-proliferative effects on colon carcinogenesis $^{(37,38)}$. Moreover, a recently published review summarised the molecular mechanisms behind the biological effects of flavanones, and concluded that flavanones may even have a broader range of biological applications in cancer 
Table 6. Colorectal cancer according to quartiles $(Q)$ of flavonoids intake from total diet by sex (Odds ratios and $95 \%$ confidence intervals)

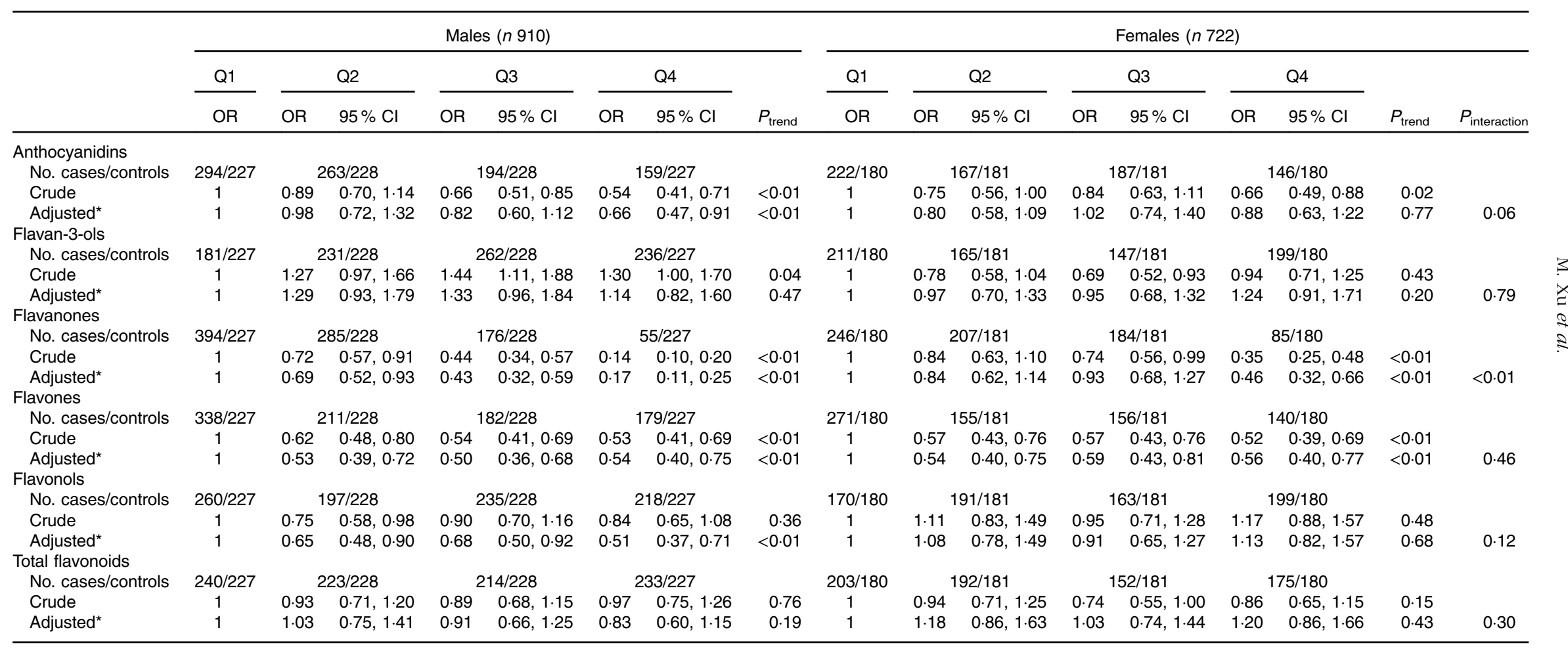

* OR was adjusted for age, sex, marital status, education, income, occupation, family history of cancer, smoking status, passive smoking, alcohol drinking, occupational activity, household and leisure-time activities, BMI, and intakes of red and processed meat, poultry and fish, total dairy products and eggs. 
Table 7. Colorectal cancer according to quartiles $(Q)$ of flavonoids intake from total diet by cancer site (Odds ratios and $95 \%$ confidence intervals)

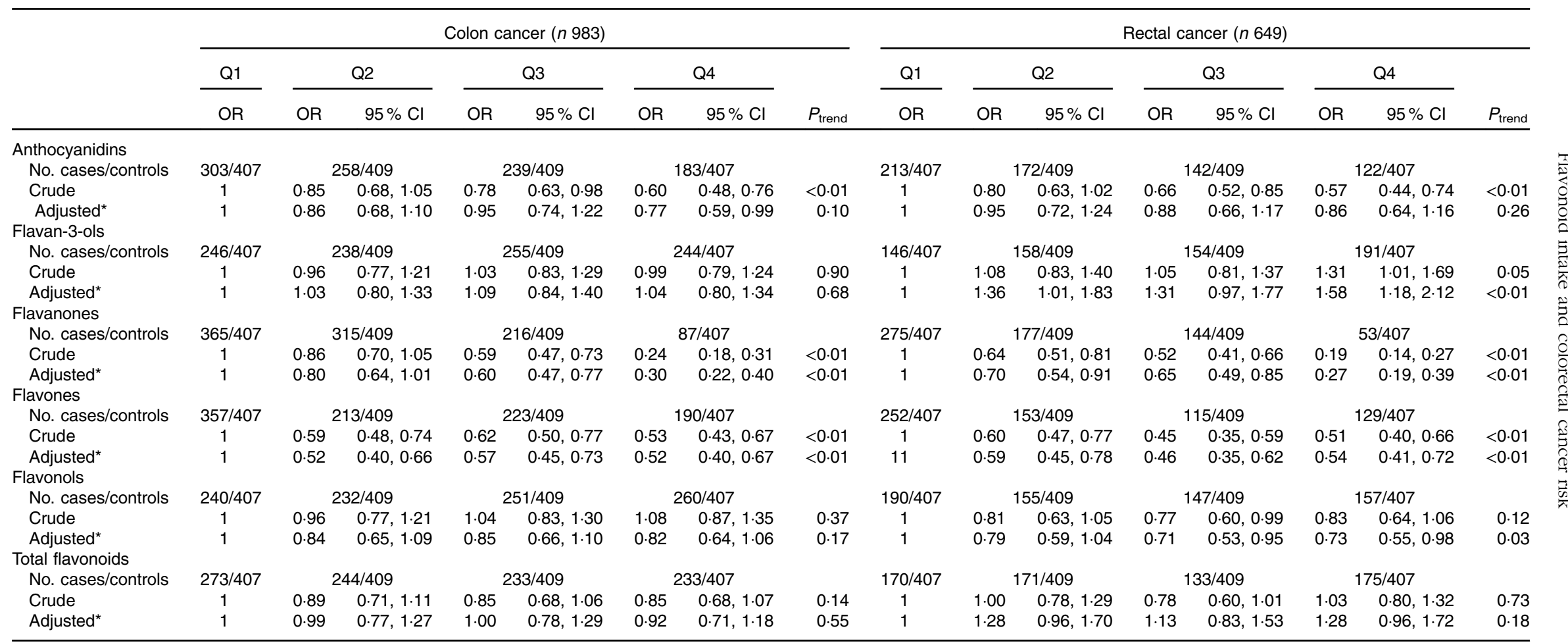

* OR was adjusted for age, sex, marital status, education, income, occupation, family history of cancer, smoking status, passive smoking, alcohol drinking, occupational activity, household and leisure-time activities, BMI, and intakes of red and processed meat, poultry and fish, total dairy products and eggs. 
Table 8. Colorectal cancer according to quartiles $(Q)$ of flavonoids intake from total diet by socio-economic status(Odds ratios and $95 \%$ confidence intervals)

\begin{tabular}{|c|c|c|c|c|c|c|c|c|c|c|c|c|c|c|c|c|}
\hline & \multicolumn{8}{|c|}{ Low socio-economic status ( $n$ 732/663) } & \multicolumn{8}{|c|}{ High socio-economic status ( $n$ 900/969) } \\
\hline & \multirow{2}{*}{$\frac{\mathrm{Q} 1}{\mathrm{OR}}$} & \multicolumn{2}{|r|}{ Q2 } & \multicolumn{2}{|r|}{ Q3 } & \multicolumn{2}{|r|}{ Q4 } & \multirow[b]{2}{*}{$P_{\text {trend }}$} & \multicolumn{2}{|l|}{ Q1 } & Q2 & \multicolumn{2}{|c|}{ Q3 } & \multicolumn{2}{|r|}{ Q4 } & \multirow[b]{2}{*}{$P_{\text {trend }}$} \\
\hline & & OR & $95 \% \mathrm{Cl}$ & OR & $95 \% \mathrm{Cl}$ & OR & $95 \% \mathrm{Cl}$ & & OR & OR & $95 \% \mathrm{Cl}$ & OR & $95 \% \mathrm{Cl}$ & OR & $95 \% \mathrm{Cl}$ & \\
\hline \multicolumn{17}{|l|}{ Anthocyanidins } \\
\hline No. cases/controls & 290/201 & \multicolumn{2}{|c|}{$175 / 163$} & \multicolumn{2}{|c|}{$152 / 148$} & \multicolumn{2}{|c|}{$115 / 151$} & & $226 / 206$ & \multicolumn{2}{|c|}{$255 / 246$} & \multicolumn{2}{|c|}{ 229/261 } & \multicolumn{2}{|c|}{$190 / 256$} & \\
\hline Crude & 1 & 0.74 & $0.56,0.98$ & 0.71 & $0.53,0.95$ & 0.53 & $0.39,0.71$ & $<0.01$ & 1 & 0.94 & $0.73,1.22$ & 0.80 & $0.62,1.04$ & 0.68 & $0.52,0.88$ & $<0.01$ \\
\hline Adjusted* & 1 & 0.71 & $0.52,0.97$ & 0.85 & $0.61,1.18$ & 0.73 & $0.51,1.03$ & 0.11 & 1 & 1.05 & $0.79,1.41$ & 1.00 & $0.74,1.34$ & 0.87 & $0.64,1.18$ & 0.31 \\
\hline \multicolumn{17}{|l|}{ Flavan-3-ols } \\
\hline No. cases/controls & $224 / 223$ & \multicolumn{2}{|c|}{$150 / 153$} & \multicolumn{2}{|c|}{$163 / 136$} & \multicolumn{2}{|c|}{$195 / 151$} & & $168 / 184$ & \multicolumn{2}{|c|}{$246 / 256$} & \multicolumn{2}{|c|}{$246 / 273$} & \multicolumn{2}{|c|}{$240 / 256$} & \\
\hline Crude & 1 & 0.98 & $0.73,1.31$ & 1.19 & $0.89,1.60$ & 1.29 & $0.97,1.70$ & 0.05 & 1 & 1.05 & $0.80,1.38$ & 0.99 & $0.75,1.29$ & 1.03 & $0.78,1.35$ & 0.96 \\
\hline Adjusted* & 1 & $1 \cdot 18$ & $0.85,1.66$ & 1.46 & $1.04,2.04$ & 1.44 & $1.04,2.00$ & 0.02 & 1 & 1.07 & $0.78,1.45$ & 0.96 & $0.71,1.30$ & 1.04 & $0.76,1.43$ & 0.98 \\
\hline \multicolumn{17}{|l|}{ Flavanones } \\
\hline No. cases/controls & $360 / 201$ & & $1 / 169$ & & 88/161 & & 3/132 & & $280 / 206$ & & $91 / 240$ & & $2 / 248$ & & 7/275 & \\
\hline Crude & 1 & 0.66 & $0.51,0.87$ & 0.44 & $0.33,0.59$ & 0.18 & $0.12,0.27$ & $<0.01$ & 1 & 0.89 & $0.70,1.14$ & 0.69 & $0.53,0.89$ & 0.26 & $0.19,0.35$ & $<0.01$ \\
\hline Adjusted* & 1 & 0.63 & $0.46,0.85$ & 0.48 & $0.35,0.67$ & 0.23 & $0.15,0.36$ & $<0.01$ & 1 & 0.92 & $0.69,1.22$ & 0.75 & $0.57,1.00$ & 0.34 & $0.24,0.47$ & $<0.01$ \\
\hline Flavones & & & & & & & & & & & & & & & & \\
\hline No. cases/controls & $335 / 213$ & & $57 / 162$ & & $30 / 146$ & & 10/142 & & $274 / 194$ & & 09/247 & & $8 / 263$ & & $99 / 265$ & \\
\hline Crude & 1 & 0.62 & $0.47,0.81$ & 0.57 & $0.42,0.76$ & 0.49 & $0.36,0.67$ & $<0.01$ & 1 & 0.60 & $0.46,0.78$ & 0.56 & $0.43,0.73$ & 0.56 & $0.43,0.72$ & $<0.01$ \\
\hline Adjusted ${ }^{*}$ & 1 & 0.55 & $0.40,0.75$ & 0.49 & $0.35,0.68$ & 0.52 & $0.37,0.72$ & $<0.01$ & 1 & 0.55 & $0.41,0.74$ & 0.57 & $0.42,0.76$ & 0.58 & $0.43,0.78$ & $<0.01$ \\
\hline Flavonols & & & & & & & & & & & & & & & & \\
\hline No. cases/controls & $230 / 191$ & & $55 / 171$ & & $2 / 152$ & & $65 / 149$ & & $200 / 216$ & & 22/238 & & 26/257 & & $52 / 258$ & \\
\hline Crude & 1 & 0.80 & $0.60,1.07$ & 0.94 & $0.70,1.26$ & 0.92 & $0.69,1.23$ & 0.75 & 1 & 1.01 & $0.77,1.31$ & 0.95 & $0.73,1.23$ & 1.05 & $0.81,1.37$ & 0.78 \\
\hline Adjusted* & 1 & 0.63 & $0.45,0.87$ & 0.78 & $0.56,1.09$ & 0.72 & $0.51,1.01$ & 0.12 & 1 & 1.01 & $0.75,1.36$ & 0.84 & $0.62,1.13$ & 0.88 & $0.65,1.18$ & 0.22 \\
\hline Total flavonoids & & & & & & & & & & & & & & & & \\
\hline No. cases/controls & $246 / 220$ & & 74/154 & & $2 / 137$ & & 70/152 & & $197 / 187$ & & $41 / 255$ & & $4 / 272$ & & $38 / 255$ & \\
\hline Crude & 1 & 1.01 & $0.76,1.34$ & 0.93 & $0.69,1.25$ & 1.00 & $0.75,1.33$ & 0.86 & 1 & 0.90 & $0.69,1.17$ & 0.78 & $0.60,1.02$ & 0.89 & $0.68,1.16$ & 0.27 \\
\hline Adjusted* & 1 & 1.32 & $0.95,1.82$ & 1.30 & $0.92,1.83$ & $1 \cdot 14$ & $0.82,1.58$ & 0.39 & 1 & 0.97 & $0.72,1.31$ & 0.86 & $0.63,1.17$ & 0.95 & $0.70,1.30$ & 0.60 \\
\hline
\end{tabular}

* OR was adjusted for age, sex, marital status, education, income, occupation, family history of cancer, smoking status, passive smoking, alcohol drinking, occupational activity, household and leisure-time activities, BMI, and intakes of red and processed meat, poultry and fish, total dairy products and eggs. 
prevention $^{(39)}$. However, intakes of subclasses of flavonoids were not associated with the risk of colorectal cancer in a recently published cohort study by Nimptsch et al. ${ }^{(15)}$. Multivariable adjusted RR comparing the highest with the lowest quintiles were 0.98 (95\% CI 0.81, 1.19) for anthocyanidins, 1.07 (95\% CI $0.95,1.21)$ for flavan-3-ols, 0.96 (95\% CI 0.84, 1.10) for flavanones, 1.01 (95\% CI $0.89,1.15)$ for flavones and 1.04 (95\% CI $0.91,1.18$ ) for flavonols. The difference could be partly explained by the variability in subclasses of flavonoid intakes among different populations. For example, the median anthocyanidins intake was about $14.6 \mathrm{mg} / \mathrm{d}$ in the US cohort ${ }^{(15,40)}$ and $20.16 \mathrm{mg} / \mathrm{d}$ in the present study. The food sources of anthocyanidins in each study were also very different. The top three food sources of anthocyanidins in the US cohort were blueberries, bananas and strawberries ${ }^{(15,40)}$, whereas anthocyanidins in the present study mainly came from eggplant, grapes and radish.

A few studies have examined the relationships between different dietary sources of flavonoid intake and colorectal cancer risk. As most of anthocyanidins, flavanones and flavones came from vegetables and fruits, the relationship between subclasses of flavonoids from vegetables and fruits was consistent with those of the total diet. However, inverse associations were found between flavan-3-ols, flavonols and total flavonoids from vegetables and fruits and colorectal cancer risk, which were not found in total diet. Consistent with the present study, a case-control study with 1163 cases and 1501 controls in the USA showed a significant protective effect of quercetin in the proximal colon when fruit intake was high or tea intake was low ${ }^{(17)}$. Another population-based, case-control study in the UK showed that flavonols and quercetin intakes from non-tea dietary sources, mainly from vegetables and fruits, were inversely associated with colorectal cancer, with OR of 0.6 (95\% CI 0.4, 1.0) for flavonols and 0.6 (95\% CI 0.4, 0.9) for quercetin $^{(18)}$. Moreover, the Iowa Women's Health Study also found that the intake of (+)-catechin and (-)-epicatechin, a subgroup of flavan-3-ols derived primarily from fruits, tended to be inversely associated with digestive tract cancer incidence ${ }^{(22)}$.

To the best of our knowledge, no previous studies have reported the relationship between tea flavonoids and colorectal cancer risk. Our study found no significant association between tea flavonoids intake and colorectal cancer risk. The percentage of tea drinkers among cases was $62.81 \%$, whereas among controls it was $54.41 \%$. This result is consistent with some previous studies ${ }^{(2)}$. However, no association was found between tea intake and colorectal cancer risk in the present study (OR 0.96; 95\% CI 0.77, 1.19, for the highest quartile $v$. the lowest). Recent findings about the association between tea intake and colorectal cancer risk may also contribute to nondetection of the significant association of tea flavonoids with colorectal cancer risk. After a median follow-up of 11.6 years, the European Investigation into Cancer and Nutrition cohort found no significant associations between tea consumption and colorectal cancer, and the RR of the highest quintile of tea intake compared with the lowest was 0.97 (95\% CI $0.86,1.09)^{(41)}$. The National Cancer Institute-Prostate, Lung, Colorectal and Ovarian Cancer Screening Trial found that greater tea intake was not associated with colorectal cancer risk
(RR 0.77; 95\% CI 0.55, 1.09 for the highest quartile $v$. the lowest) ${ }^{(42)}$. Two meta-analyses summarised the results of case-control studies and prospective studies and found that data were insufficient to conclude that green tea may protect against colorectal cancer ${ }^{(43,44)}$.

The present study showed an inverse association between colorectal cancer risk and flavonoids intake from vegetables and fruits but not from tea. One potential reason might be that the flavonoid glycosides vary among different dietary sources and different compounds of glycosides have different bioavailability. Previous studies have shown that onions contain glucose conjugates of quercetin, apples contained both glucose- and non-glucose quercetin glycosides and the major quercetin glycoside in tea is pure quercetin-3-rutinoside ${ }^{(45)}$. Comparing the bioavailability of flavonol quercetin from major dietary sources in human subjects found that quercetin glucosides from onions are absorbed rapidly, and the rutinoside in tea is absorbed slowly, whereas the overall absorption rate of various glycosides from apples is intermediate ${ }^{(45)}$. In addition, other compounds such as dietary fibre, carotenoids and vitamins present in vegetables and fruits might be responsible for the beneficial effects. Some studies have shown that intakes of dietary fibre, carotenoids and vitamins from vegetables and fruits are inversely associated with colorectal cancer risk ${ }^{(46-48)}$.

Flavonoids, especially from vegetables and fruits, have several important biological functions, which might be related to cancer risk ${ }^{(5)}$. Isorhamnetin diglucoside, a major flavonoid present in mustard leaf, was found to be metabolised to isorhamnetin in vivo by intestinal bacteria and plays an important role as an antioxidant ${ }^{(49)}$. It has been shown that flavan-3-ols from apples can reactivate silenced tumour suppressor genes in colorectal cancer cells ${ }^{(50)}$. Another study demonstrated that a standardised berry anthocyanin-rich extract inhibited the proliferation of the Caco- 2 human colorectal cancer cell line by promoting reactive oxygen species accumulation, inducing caspase-3 activation, and by up-regulating the expression of $p 21 \mathrm{Waf} / \mathrm{Cif1} \mathrm{1}^{(51)}$. Moreover, in vitro and in vivo studies have also shown that flavonoids can influence signal transduction pathways, stimulate apoptosis, and inhibit inflammation and proliferation in human cancer cell lines ${ }^{(5,6)}$. In addition, substantial amounts reach the colon, where they are further metabolised into metabolites that may also mediate some biological activity ${ }^{(52)}$.

The present study showed no interaction between flavonoids intakes and sex on the risk of colorectal cancer. The inverse association between intakes of anthocyanidins and flavonols and colorectal cancer risk observed among men but not among women in the present study might be a chance finding. Moreover, no previous studies have reported that different hormone status between men and women could affect the bioavailability of flavonoids ${ }^{(10,15)}$. Subgroup analysis by cancer site showed that the protective effect exerted by total anthocyanidins, flavanones and flavones intakes on colon, rectal and combined cancers was similar, in agreement with other studies ${ }^{(16,20,21)}$.

At least two strengths were present in this study. First, a few studies have examined the associations between flavonoids from different dietary sources and the risk of colorectal cancer. Flavonoids intake from vegetables and fruits may exert more 
protective effect on colorectal cancer risk than tea flavonoids. Therefore, it is necessary to detect the association between flavonoids from different dietary sources and colorectal cancer in future studies. Second, compared with previous case-control studies, the present study had a relatively large sample size. We have enough power to detect small associations in risk of colorectal cancer.

There are several limitations that should be taken into account when interpreting the results. First, selection bias is a potential limitation in case-control studies. To minimise selection bias, we tried to recruit controls from different departments of three general hospitals with ailments that had no apparent association with dietary habits. Moreover, the relatively high participation rate $(89.57 \%$ for cases and $86.98 \%$ for hospital-derived controls, respectively) in the present study also reduced the selection bias. Furthermore, although cases from Sun Yat-sen University Cancer Center had greater household income and lower education levels than controls recruited from the community and other hospitals, no interaction effect was found between socio-economic status and flavonoids intake. Therefore, the possibility of selection bias should be reduced in our study. Second, dietary habits can be influenced by a recent diagnosis of cancer or by disease progress, so we tried to interview the cases as soon as the diagnosis was made. The average interval between diagnosis and interview for cases was $11 \mathrm{~d}$. Third, the potential for recall bias exists in our study, as in any case-control study. To minimise this bias, a standardised questionnaire interview method was used to improve the comparability of recall between cases and controls. We also provided photographs of foods with usual portion size to help participants accurately estimate food intake. Fourth, although food intake was assessed only once during the past 12-month period, dietary assessment using the validated FFQ can be representative of long-term dietary habits ${ }^{(34)}$. In addition, random measurement error in the estimation of dietary flavonoids intake using FFQ is also of concern. However, usually, this misclassification is non-differential among cases and controls ${ }^{(53)}$ and would attenuate the true association between flavonoids intake and colorectal cancer risk. Finally, despite the fact that we adjusted for a number of potential confounders, the possibility of residual confounding may have affected the results.

In conclusion, the results from this relatively large case-control study suggest that intakes of total anthocyanidins, flavanones and flavones may decrease the risk of colorectal cancer. In addition, the inverse association between colorectal cancer risk and flavonoids from vegetables and fruits, but not tea flavonoids, suggest the importance of assessing independent associations between different dietary sources of flavonoids and disease risk.

\section{Acknowledgements}

The authors gratefully acknowledge the cooperation of the study participants.

This study was supported by Guangdong Natural Science Foundation (No.: 2014A030313188, 2016A030313225). The funders had no role in the design, analysis or writing of this article.

The authors' responsibilities were as follows: M. X. collected the data, analysed the data and wrote the paper. Y.-M. C. provided significant advice regarding the analyses and interpretation of the data. J. H., W.-Q. H., B. Y. and M.-S. L. participated in data collection. Y.-J. F. and Z.-Z. P. were responsible for connecting and coordinating the field work. C.-X. Z constructed the project design, supervised the study and contributed to manuscript writing.

None of the authors has any conflicts of interest to declare.

\section{References}

1. van Duijnhoven FJ, Bueno-De-Mesquita $\mathrm{HB}$, Ferrari $\mathrm{P}$, et al. (2009) Fruit, vegetables, and colorectal cancer risk: the European Prospective Investigation into Cancer and Nutrition. Am J Clin Nutr 89, 1441-1452.

2. Green CJ, de Dauwe P, Boyle T, et al. (2014) Tea, coffee, and milk consumption and colorectal cancer risk. J Epidemiol 24, 146-153.

3. Bradbury KE, Appleby PN \& Key TJ (2014) Fruit, vegetable, and fiber intake in relation to cancer risk: findings from the European Prospective Investigation into Cancer and Nutrition (EPIC). Am J Clin Nutr 100, Suppl. 1, S394-S398.

4. Aherne SA \& O'Brien NM (2002) Dietary flavonols: chemistry, food content, and metabolism. Nutrition 18, 75-81.

5. Pierini R, Gee JM, Belshaw NJ, et al. (2008) Flavonoids and intestinal cancers. Br J Nutr 99, Suppl. 1, S53-S59.

6. Nichenametla SN, Taruscio TG, Barney DL, et al. (2006) A review of the effects and mechanisms of polyphenolics in cancer. Crit Rev Food Sci Nutr 46, 161-183.

7. Kocic B, Kitic D \& Brankovic S (2013) Dietary flavonoid intake and colorectal cancer risk: evidence from human population studies. $J$ BUON 18, 34-43.

8. Woo HD \& Kim J (2013) Dietary flavonoid intake and risk of stomach and colorectal cancer. World J Gastroenterol 19 , 1011-1019.

9. Jin H, Leng Q \& Li C (2012) Dietary flavonoid for preventing colorectal neoplasms. The Cochrane Database of Systematic Reviews, issue 8, D9350. http://onlinelibrary.wiley.com/doi/ 10.1002/14651858.CD009350.pub2/full

10. Lin J, Zhang SM, Wu K, et al. (2006) Flavonoid intake and colorectal cancer risk in men and women. Am J Epidemiol 164, 644-651.

11. Simons CC, Hughes LA, Arts IC, et al. (2009) Dietary flavonol, flavone and catechin intake and risk of colorectal cancer in the Netherlands Cohort Study. Int J Cancer 125, $2945-2952$

12. Wang L, Lee IM, Zhang SM, et al. (2009) Dietary intake of selected flavonols, flavones, and flavonoid-rich foods and risk of cancer in middle-aged and older women. Am J Clin Nutr 89, 905-912.

13. Mursu J, Nurmi T, Tuomainen TP, et al. (2008) Intake of flavonoids and risk of cancer in Finnish men: the Kuopio Ischaemic Heart Disease Risk Factor Study. Int J Cancer 123, 660-663.

14. Cutler GJ, Nettleton JA, Ross JA, et al. (2008) Dietary flavonoid intake and risk of cancer in postmenopausal women: the Iowa Women's Health Study. Int J Cancer 123, 664-671.

15. Nimptsch K, Zhang X, Cassidy A, et al. (2016) Habitual intake of flavonoid subclasses and risk of colorectal cancer in 2 large prospective cohorts. Am J Clin Nutr 103, 184-191. 
16. Zamora-Ros R, Not C, Guino E, et al. (2013) Association between habitual dietary flavonoid and lignan intake and colorectal cancer in a Spanish case-control study (the Bellvitge Colorectal Cancer Study). Cancer Causes Control 24, 549-557.

17. Djuric Z, Severson RK \& Kato I (2012) Association of dietary quercetin with reduced risk of proximal colon cancer. Nutr Cancer 64, 351-360.

18. Kyle JA, Sharp L, Little J, et al. (2010) Dietary flavonoid intake and colorectal cancer: a case-control study. Br J Nutr $\mathbf{1 0 3}$, 429-436.

19. Rossi M, Bosetti C, Negri E, et al. (2010) Flavonoids, proanthocyanidins, and cancer risk: a network of case-control studies from Italy. Nutr Cancer 62, 871-877.

20. Theodoratou E, Kyle J, Cetnarskyj R, et al. (2007) Dietary flavonoids and the risk of colorectal cancer. Cancer Epidemiol Biomarkers Prev 16, 684-693.

21. Rossi M, Negri E, Talamini R, et al. (2006) Flavonoids and colorectal cancer in Italy. Cancer Epidemiol Biomarkers Prev 15, 1555-1558

22. Arts IC, Jacobs DJ, Gross M, et al. (2002) Dietary catechins and cancer incidence among postmenopausal women: the Iowa Women's Health Study (United States). Cancer Causes Control 13, 373-382.

23. Zamora-Ros R, Knaze V, Lujan-Barroso L, et al. (2013) Differences in dietary intakes, food sources and determinants of total flavonoids between Mediterranean and nonMediterranean countries participating in the European Prospective Investigation into Cancer and Nutrition (EPIC) study. Br J Nutr 109, 1498-1507.

24. Arts IC, Hollman PC, Bueno DMH, et al. (2001) Dietary catechins and epithelial cancer incidence: the Zutphen elderly study. Int J Cancer 92, 298-302.

25. Hall JN, Moore S, Harper SB, et al. (2009) Global variability in fruit and vegetable consumption. Am J Prev Med 36, 402-409.

26. Luo WP, Fang YJ, Lu MS, et al. (2015) High consumption of vegetable and fruit colour groups is inversely associated with the risk of colorectal cancer: a case-control study. Br J Nutr 113, 1129-1138.

27. Lu MS, Fang YJ, Pan ZZ, et al. (2015) Choline and betaine intake and colorectal cancer risk in Chinese population: a case-control study. PLOS ONE 10, e118661.

28. Zhong X, Fang YJ, Pan ZZ, et al. (2013) Dietary fat, fatty acid intakes and colorectal cancer risk in Chinese adults. Eur J Cancer Prev 22, 438-447.

29. World Health Organization (1998) Guidelines for Controlling and Monitoring the Tobacco Epidemic. Geneva: WHO.

30. Ainsworth BE, Haskell WL, Herrmann SD, et al. (2011) 2011 Compendium of Physical Activities: a second update of codes and MET values. Med Sci Sports Exerc 43, 1575-1581.

31. Ainsworth BE, Haskell WL, Whitt MC, et al. (2000) Compendium of Physical Activities: an update of activity codes and MET intensities. Med Sci Sports Exerc 32, S498-S504.

32. Yang YX, Wang GY \& Pan XC (2002) China Food Composition. Beijing: Peking University Medical Press.

33. Bhagwat S, Haytowitz DB \& Holden JM (2014) USDA Database for the Flavonoid Content of Selected Foods Release 3.1. Beltsville, MD: USDA.

34. Zhang CX \& Ho SC (2009) Validity and reproducibility of a food frequency questionnaire among Chinese women in Guangdong province. Asia Pac J Clin Nutr 18, 240-250.

35. Willett WC, Howe GR \& Kushi LH (1997) Adjustment for total energy intake in epidemiologic studies. Am J Clin Nutr $\mathbf{6 5}$, S1220-S1228; S1229-S1231.

36. Zamora-Ros R, Knaze V, Romieu I, et al. (2013) Impact of thearubigins on the estimation of total dietary flavonoids in the European Prospective Investigation into Cancer and Nutrition (EPIC) study. Eur J Clin Nutr 67, 779-782.

37. Saiprasad G, Chitra P, Manikandan R, et al. (2014) Hesperidin induces apoptosis and triggers autophagic markers through inhibition of Aurora-A mediated phosphoinositide-3-kinase/ Akt/mammalian target of rapamycin and glycogen synthase kinase-3 beta signalling cascades in experimental colon carcinogenesis. Eur J Cancer 50, 2489-2507.

38. Song HM, Park GH, Eo HJ, et al. (2015) Anti-proliferative effect of naringenin through p38-dependent downregulation of cyclin D1 in human colorectal cancer cells. Biomol Ther (Seoul) 23, 339-344.

39. Roohbakhsh A, Parhiz H, Soltani F, et al. (2015) Molecular mechanisms behind the biological effects of hesperidin and hesperetin for the prevention of cancer and cardiovascular diseases. Life Sci 124, 64-74.

40. Cassidy A, O'Reilly EJ, Kay C, et al. (2011) Habitual intake of flavonoid subclasses and incident hypertension in adults. Am J Clin Nutr 93, 338-347.

41. Dik VK, Bueno-de-Mesquita HB, Van Oijen MG, et al. (2014) Coffee and tea consumption, genotype-based CYP1A2 and NAT2 activity and colorectal cancer risk-results from the EPIC cohort study. Int J Cancer 135, 401-412.

42. Dominianni C, Huang WY, Berndt S, et al. (2013) Prospective study of the relationship between coffee and tea with colorectal cancer risk: the PLCO Cancer Screening Trial. $\mathrm{Br} J$ Cancer 109, 1352-1359.

43. Wang XJ, Zeng XT, Duan XL, et al. (2012) Association between green tea and colorectal cancer risk: a meta-analysis of 13 case-control studies. Asian Pac J Cancer Prev 13, 3123-3127.

44. Wang ZH, Gao QY \& Fang JY (2012) Green tea and incidence of colorectal cancer: evidence from prospective cohort studies. Nutr Cancer 64, 1143-1152.

45. Hollman PC \& Katan MB (1999) Dietary flavonoids: intake, health effects and bioavailability. Food Chem Toxicol 37, 937-942.

46. Lu MS, Fang YJ, Chen YM, et al. (2015) Higher intake of carotenoid is associated with a lower risk of colorectal cancer in Chinese adults: a case-control study. Eur J Nutr $\mathbf{5 4}$, 619-628.

47. Zhong X, Fang YJ, Pan ZZ, et al. (2014) Dietary fiber and fiber fraction intakes and colorectal cancer risk in Chinese adults. Nutr Cancer 66, 351-361.

48. Byers T \& Guerrero N (1995) Epidemiologic evidence for vitamin $\mathrm{C}$ and vitamin $\mathrm{E}$ in cancer prevention. Am J Clin Nutr 62, S1385-S1392.

49. Yokozawa T, Kim HY, Cho EJ, et al. (2002) Antioxidant effects of isorhamnetin 3,7-di-O-beta-D-glucopyranoside isolated from mustard leaf (Brassica juncea) in rats with streptozotocin-induced diabetes. J Agric Food Chem 50, 5490-5495.

50. Fini L, Selgrad M, Fogliano V, et al. (2007) Annurca apple polyphenols have potent demethylating activity and can reactivate silenced tumor suppressor genes in colorectal cancer cells. J Nutr 137, 2622-2628.

51. Anwar S, Fratantonio D, Ferrari D, et al. (2016) Berry anthocyanins reduce proliferation of human colorectal carcinoma cells by inducing caspase- 3 activation and p21 upregulation. Mol Med Rep 14, 1397-1403.

52. Williamson G \& Clifford MN (2010) Colonic metabolites of berry polyphenols: the missing link to biological activity? $\mathrm{Br} \mathrm{J}$ Nutr 104, Suppl. 3, S48-S66.

53. Kipnis V, Midthune D, Freedman LS, et al. (2001) Empirical evidence of correlated biases in dietary assessment instruments and its implications. Am J Epidemiol 153, 394-403. 